\title{
193. NOVEDADES FLORÍSTICAS PARA ANDALUCÍA (SUR DE ESPAÑA)
}

\author{
Gabriel BLANCA $^{1 *}$, Miguel CUETO², Julián FUENTES CARRETERO ${ }^{3}$ \\ y Leonardo GUTIÉRREZ CARRETERO ${ }^{4}$
}

Recibido el 4 de noviembre de 2014, aceptado para su publicación el 6 de octubre de 2015

New records for the flora of Andalusia (South Spain)

Palabras clave. Flora vascular, corología, Andalucía, España.

Key words. Vascular flora, chorology, Andalusia, Spain.

Como resultado de recientes campañas de herborización en Andalucía Oriental, se aportan 6 citas que suponen novedades o confirmación para el conjunto de la flora andaluza.

Se han tomado como obras de referencia la Flora Vascular de Andalucía Occidental (Valdés et al., 1987), la Flora Vascular de Andalucía Oriental (Blanca et al., 2009, 2011) y Flora Iberica (Castroviejo, 1986-2013). El material recolectado está depositado en los herbarios de la Universidad de Granada (GDA) y de la Universidad de Almería (HUAL).

\section{Ipomoea cairica (L.) Sweet}

ESPAÑA. Almería. La Cañada de San Urbano, crtra. de acceso al aparcamiento norte de la Universidad, $5 \mathrm{~m}$, bordes de antiguos cultivos, sobre Phoenix dactylifera, 25-8-2014, M. Cueto (HUAL 25229). Granada. Vélez Benaudalla, Azud de Vélez, 50 m, naturalizada en setos, 10-10-2014, G. Blanca \& M. Cueto (GDA 61380).

Novedad para la flora andaluza. Especie cultivada de indudable valor ornamental, originaria de zonas tropicales de Asia y África (Sánchez de Lorenzo-Cáceres, 2010) y actualmente extendida por gran parte del Mundo. No aparece citada en ninguna de las obras de referencia de Andalucía (Valdés et al., 1987; Blanca et al., 2009, 2011). Tampoco Silvestre (2012) la incluyó entre las especies ibéricas, aunque recogió la cita de Serra Laliga (2007) en Alicante. Se encuentra naturalizada formando masas de gran densidad, en sitios húmedos (borde de acequias, arroyuelos, etc.). Según la terminología propuesta por Sanz Elorza et al. (2004), se trata de un metáfito epecófito.

\section{Matricaria discoidea DC.}

ESPAÑA. Granada. 30SVG6505, Monachil, Sierra Nevada, Albergue Universitario, $2520 \mathrm{~m}$, pastizales terofíticos nitrificados, desarrollados sobre sustrato silíceo, 29-8-2013, J. Vilches (GDA 59950).

Novedad para la flora andaluza. Originaria del noreste de Asia, naturalizada en la mayor parte de Europa (Kay, 1976). Localmente abundante en los alrededores del Albergue Universitario (estación de Solynieve, Sierra Nevada, Granada), donde es muy probable que se haya naturalizado recientemente a partir del material utilizado para las infusiones que preparan los quioscos situados en las inmediaciones. En la actualidad no representa ninguna amenaza para la flora autóctona, pues solo se localiza en una zona ya de por sí fuertemente perturbada de la estación invernal. Según la terminología propuesta por Sanz 
Elorza et al. (2004), se trata de un metáfito epecófito.

\section{Phalaris arundinacea L.}

ESPAÑA. Jaén. Sierra de Segura, río Madera, bordes del río, sustrato calizo, 13-9-2013, G. Blanca, M. Cueto \& J. Fuentes (GDA 60128). 30SWH3825, Sierra de Segura, La Toba, Río Segura, 965 m, bordes de río y peñascos dentro del propio curso de agua, 15-7-2014, J. Fuentes (GDA 61227). 30S 0534596 4224466, Río Segura a Poyotello, 995 m, borde del río, 8-8-2012, M. Cueto \& G. Blanca (HUAL 25249).

Se confirma la presencia de esta especie holártica (Bolòs \& Vigo, 2001) en Andalucía (Sierra de Segura, Jaén). Fue citada por primera vez en la misma serranía, más concretamente en Santiago de la Espada, ribera del río Segura, por

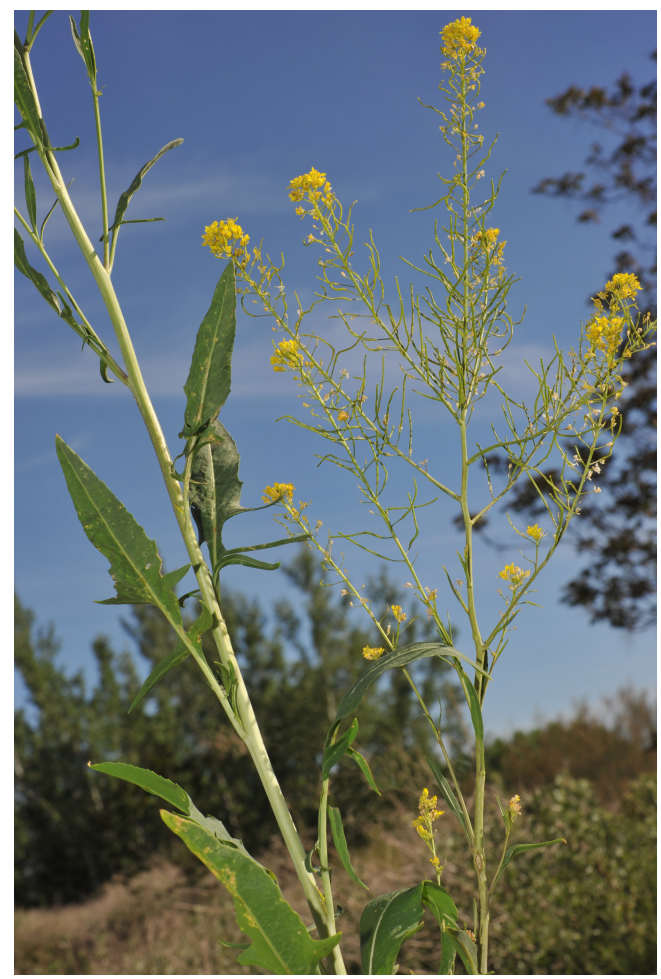

Figura 1. Sisymbrium assoanum Loscos \& Pardo
Soriano Martín (1984), aunque posteriormente no se incluyó en la flora de Andalucía Oriental (Romero, 2009). Su presencia se limita al borde de ríos y a pequeños islotes situados en el interior del propio cauce, donde convive con Salix spp., Carex spp., Festuca fenas, Epipactis palustris, Teucrium scordium, Parnassia palustris, Sanguisorba officinalis, Lycopus europaeus, etc.

\section{Rorippa palustris (L.) Besser}

ESPAÑA. Granada. Embalse del Cubillas, terrenos margosos de la plataforma de inundación del embalse, 31/08/2015, G. Blanca (GDA 62004). Granada. 30SVG42, Embalse del Cubillas, Albolote, $650 \mathrm{~m}$, terrenos margosos de la plataforma de inundación del embalse, 16/09/2015, M. Cueto \& G. Blanca (HUAL 25940).

Euroasiática y Norte de América, naturalizada en América Central y del Sur. Citada de la mitad norte de la Península Ibérica (Martínez 1986), con referencias posteriores en Barcelona (Sáez et al. 2010) y Navarra (Campos et al. 2003). Novedad para la flora andaluza, la población está formada por 50-100 individuos establecidos sobre los bloques de limos cuarteados por la falta de agua así como en los bordes de comunidades de Xanthium strumarium L.

Sisymbrium assoanum Loscos \& Pardo (fig. 1)

ESPAÑA. Granada. 30SWF3978, Hoya de Baza, Galera, carretera A-330, 860 m, taludes y arcenes de margas yesíferas, subnitrófila, 1-4-2014, G. Blanca \& J. Fuentes (GDA 61204).

Endemismo del C y E de la Península Ibérica, desde la provincia de Huesca hasta la de Albacete (Pujadas Salvá, 1993). Novedad para la flora andaluza. La nueva localidad es la más meridional conocida para la especie; está formada por más de un centenar de individuos, algunos de gran desarrollo (más de $1 \mathrm{~m}$ de 
altura), asentados en taludes próximos al río Galera.

\section{Verbascum thapsus L.}

ESPAÑA. Granada. Puebla de Don Fadrique, Nablanca, $1500 \mathrm{~m}$, cunetas de la carretera, 1-8-2014, M. Cueto \& G. Blanca (HUAL 25240).

Especie de distribución euroasiática, que alcanza principalmente la mitad $\mathrm{N}$ de la Península Ibérica (Benedí, 2009). Novedad para la flora andaluza. Las poblaciones andaluzas de esta especie han sido asignadas tradicionalmente a otra especie próxima, $V$. giganteum Willk., de la que se distingue por su indumento verdoso, pecíolos más estrechos (3-7 $\mathrm{mm}$ de anchura) ni amplexicaules ni dilatados en la base, brácteas más pequeñas [6-9(15) mm], y flores más pequeñas (sépalos 5-7 mm, corola 18-23 $\mathrm{mm}$ ) (Benedí, l.c.). En Andalucía es frecuente en la Sierra de Guillimona (Granada), a la que pertenece la localidad estudiada, y en el macizo de Segura-Cazorla (Jaén), donde se ha observado viviendo en márgenes de caminos y carreteras, sobre sustrato calizo.

AGRADECIMIENTOS. A J. Vilches por la información sobre Matricaria discoidea.

\section{BIBLIOGRAFÍA}

BENEDÍ, C. -2009- Verbascum L. En: C. Benedí, E. Rico, J. Güemes \& A. Herrero -eds- Flora Iberica 13: 49-97. Real Jardín Botánico. CSIC. Madrid.

BLANCA, G., B. CABEZUDO, M. CUETO, C. FERNÁNDEZ LÓPEZ \& C. MORALES TORRES -2009, eds.- Flora Vascular de Andalucía Oriental. Consejería de Medio Ambiente, Junta de Andalucía. Sevilla.

BLANCA, G., B. CABEZUDO, M. CUETO, C. MORALES TORRES \& C. SALAZAR -2011, eds.- Flora Vascular de Andalucía Oriental. Universidades de Almería, Granada, Jaén y Málaga. Granada.
BOLÒS, O. de \& J. VIGO -2001- Flora dels Països Catalans. Volum IV (Monocotiledònies). Editorial Barcino. Barcelona.

CAMPOS, J.A., DARQUESTADE, A., BIURRUN, I. \& I. GARCÍA MIJANGOS -2003- Sobre algunas plantas poco conocidas del País Vasco y zonas limítrofes (II). Est. Mus. Cienc. Nat. de Álava 18-19: 59-68.

CASTROVIEJO, S. -1986-2013, coord.- Flora Iberica. Real Jardín Botánico. CSIC. Madrid.

KAY, Q.O.N. -1976- Chamomilla S.F. Gray. In: T.G. Tutin, V.H. Heywood, N.A. Burges, D.M. Moore, D.H. Valentine, S.M. Walters \& D.A. Webb -eds.- Flora Europaea 4: 167. Cambridge University Press. Cambridge.

MARTÍNEZ LABORDE, J.B. -1993- Rorippa Scop. En: S. Castroviejo, C. Aedo, C. Gómez Campo, M. Laínz, P. Montserrat, R. Morales, F. Muñoz Garmendia, G. Nieto Feliner, E. Rico, S. Talavera \& L. Villar-eds.- Flora Iberica 4: 106-117. Real Jardín Botánico. CSIC. Madrid.

PUJADAS SALVÁ, A. -1993- Sisymbrium L. En: S. Castroviejo, C. Aedo, C. Gómez Campo, M. Laínz, P. Montserrat, R. Morales, F. Muñoz Garmendia, G. Nieto Feliner, E. Rico, S. Talavera \& L. Villar-eds.- Flora Iberica 4: 12-27. Real Jardín Botánico. CSIC. Madrid.

ROMERO, A.T. (2009). Phalaris L. En: G. Blanca, B. Cabezudo, M. Cueto, C. Fernández López \& C. Morales Torres -eds.- Flora Vascular de Andalucía Oriental 1: 362-364. Consejería de Medio Ambiente, Junta de Andalucía. Sevilla.

SÁEZ, L., AYMERICH, P. \& C. BLANCHÉ -2010- Llibre vermell de les plantes vasculars endèmiques i amenaçades de Catalunya. Argania editio.

SÁNCHEZ DE LORENZO-CÁCERES, J.M. -2010Convolvulaceae. En: J.M. Sánchez de LorenzoCáceres -coord.- Flora Ornamental Española. VI. Araliaceae-Boraginaceae, págs. 380-399. Junta de Andalucía, Grupo Mundi-Prensa \& Asociación Española de Parques y Jardines Públicos, Madrid.

SANZ ELORZA, M., E.D. DANA SÁNCHEZ \& E. SOBRINO VESPERINAS -2004, eds.- Atlas de las Plantas Alóctonas Invasoras en España. Dirección General para la Biodiversidad. Madrid.

SERRA LALIGA, L. -2007- Estudio crítico de la flora vascular de la provincia de Alicante: 
aspectos nomenclaturales, biogeográficos y de conservación. Ruizia 19. Real Jardín Botánico. CSIC. Madrid.

SILVESTRE, S. -2012- Ipomoea L. En: S. Talavera, C. Andrés, M. Arista, M.P. Fernández Piedra, M.J. Gallego, P.L. Ortiz, C. Romero Zarco, F.J. Salgueiro, S. Silvestre \& A. Quintanar -eds.Flora Iberica 11:279-286. Real Jardín Botánico. CSIC. Madrid.

SORIANO MARTÍN, C. -1984- Contribución al catálogo florístico del macizo de Segura-Cazorla (Andalucía, España), III. Fontqueria 5: 33-34.
VALDÉS, B., S. TALAVERA \& E. FERNÁNDEZGALIANO -1987- Flora Vascular de Andalucía Occidental. Ketres editora. Barcelona.

Dirección de los autores. ${ }^{1}$ Departamento de Botánica. Facultad de Ciencias. Universidad de Granada. 18001 Granada. ${ }^{2}$ Departamento de Biología y Geología, Universidad de Almería. 04120 Almería. ${ }^{3} \mathrm{C} /$ Castillo 5, Bajo F. 18152 La Zubia (Granada). ${ }^{4}$ Jardín Botánico Umbría de la Virgen. Consejería de Medio Ambiente y Ordenación del Territorio, Junta de Andalucía. 04838 María (Almería). "Autor para correspondencia: gblanca@ugr.es.

194. CITAS NUEVAS O INTERESANTES PARA LA FLORA VASCULAR DE SIERRA NEVADA Y SIERRA DE BAZA (GRANADA, ESPAÑA)

Julián Manuel FUENTES CARRETERO ${ }^{1 *}$ \& Miguel CUETO $^{2}$

Recibido el 1 octubre de 2015, aceptado para su publicación el 20 de octubre de 2015

New or interesting locations for vascular flora of Sierra Nevada and Sierra de Baza (Granada, Spain).

Palabras clave. Plantas raras, corología, localidades nuevas, Sureste ibérico, Andalucía.

Key words. Rare plants, chorology, new records, South-eastern Iberian Peninsula, Andalusia.

En los macizos de Sierra Nevada y Sierra de Baza (esta última muy cercana a la anterior) habita una flora de gran interés por su variedad, carácter endémico y relicto. Engloban algunos territorios legalmente protegidos como el Parque Nacional de Sierra Nevada y parte de los parques naturales de Sierra Nevada y Sierra de Baza. Este trabajo contribuye al conocimiento de la flora de estas dos sierras.

La nomenclatura empleada, así como su verificación corológica, es la establecida por Blanca \& Morales (1991), la Flora Vascular de Andalucía Oriental (Blanca et al., 2011) y Flora Ibérica (Castroviejo et al., 1986-2014), además de otras obras referenciadas en el texto. Para establecer el estado de conservación se han utilizado las propuestas de Cabezudo et al. (2005) y Moreno (2008). El material recolectado se encuentra depositado en los herbarios de las Universidades de Granada 